\title{
Estudos sociais em ciência e tecnologia e suas distintas abordagens
}

ADRIANO PREMEBIDA"

FABRÍCIO MONTEIRO NEVES"

JALCIONE ALMEIDA

\section{Resumo}

Esta breve introdução aos Estudos Sociais em Ciência e Tecnologia (ESCT) propõe expor os temas mais correntes e as distintas abordagens teóricas que fazem parte deste campo ainda novo de pesquisa. Busca-se fornecer um painel das disputas no exterior do campo, entre sociólogos e epistemólogos, e, no interior do campo, entre as distintas perspectivas sobre ciência e tecnologia que hoje ocupam sociólogos, antropólogos e cientistas políticos na composição deste rico cenário de pesquisa social.

Palavras-chave: Conhecimento. Ciência. Tecnologia. Simetria.

\footnotetext{
* Pesquisador e Diretor Científico da Fundação Djalma Batista (FDB), Manaus. premebida@ hotmail.com

** Professor da Universidade Federal de Santa Maria (UFSM), Departamento de Ciências Sociais.fabriciomneves@yahoo.com.br

*** Professor da Universidade Federal do Rio Grande do Sul (UFRGS), Departamento de Sociologia e Programa de Pós-Graduação em Sociologia. Pesquisador CNPq. jal@ufrgs.br
} 
Sociologias, Porto Alegre, ano 13, no 26, jan./abr. 2011, p. 22-42

\section{Introdução}

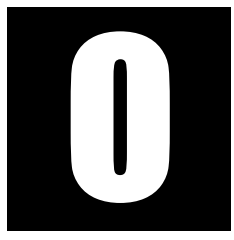

s estudos sociais em ciência e tecnologia (ESCT), caracterizados pela união de diversas abordagens e metodologias das ciências sociais são ainda incipientes no Brasil, a despeito de décadas de pesquisa nos Estados Unidos e Europa. Somente nos últimos anos surgem programas de pós-graduação, periódicos e congressos especializados nestas abordagens e temas. Além disso, pela trajetória histórica e lugar periférico ocupado pela ciência e tecnologia na sociedade brasileira, tais estudos ainda são muito focados em políticas públicas de ciência e tecnologia e com abordagens teóricas limitadas, do ponto de vista dos resultados dos estudos empíricos.

Este artigo tem como proposta aduzir, sinteticamente, a alguns elementos teóricos e metodológicos desta grande área de estudo, no sentido de dar suporte aos textos que formam este dossiê. Os ESCT ou Estudos em Ciência, Tecnologia e Sociedade (CTS), em sua multiplicidade de abordagens, têm sua unidade no esforço de compreender objetos de estudo cada vez mais destacáveis na sociedade contemporânea, a saber, a ciência e a tecnologia. Tais objetos se ramificam em muitos outros como: investigações acerca dos condicionantes sociais da estruturação e autonomia do campo científico; formação do conteúdo científico e tecnológico e sua compleição institucional, de acordo com diferenças culturais e regionais; relações com o complexo industrial e a caracterização do consumo contemporâneo das inovações tecnológicas; formas de decisão e escolhas sobre os grandes sistemas técnicos especializados que gerenciam a vida cotidiana; relação entre peritos e leigos no contexto de produção e difusão destes conhecimentos; mecanismos e condições institucionais e sociais de estruturação da ciência e tecnologia, por região e suas diferenças socioculturais; mecanismos de engajamento público nos temas 
sociotécnicos e; estudos sobre os impactos socioambientais decorrentes da utilização de sistemas e artefatos tecnológicos no dia-a-dia.

A argumentação que se seguirá pretende mostrar a prática científica como uma ação para além da pura contemplação humana na apreensão do mundo em seu caráter material e abstrato, algo muito diferente do que aparece em muitos livros-texto de cursos de graduação. A ciência envolve uma socialização, uma execução rotineira de aprendizado formal e informal. Ela exprime uma ativa diligência sobre substâncias, seres vivos, moléculas, proteínas, compostos químicos e artefatos técnicos, não apenas em um reflexo da realidade tal como é, mas construindo-a através de relatos provisórios com a ajuda de máquinas e equipamentos de análise e organização de dados. O laboratório, de acordo com algumas abordagens sociológicas (LATOUR; WOOLGAR, 1997 e LYNCH, 1985), é o local privilegiado no estudo desta ação. É neste ambiente que aspectos teóricos e epistemológicos das disciplinas científicas tomam forma mais acabada, mas também a rotina e o conhecimento tácito experimental são incorporados nas novas gerações de engenheiros, técnicos e cientistas através de aprendizado formal, vivência linguística e produção de uma narrativa sobre a realidade, através da correlação de teorias, conceitos, hipóteses, gráficos e textos.

\section{Breve histórico e abordagens clássicas}

Esta área de estudos tem seu período de emergência histórica no final da segunda metade do século XIX, influenciada por alguns filósofos que se depararam com o problema dos elementos geradores e decisivos na construção, sustentação e transformação do conhecimento. Estes problemas são primeiramente analisados de um ponto de vista mais filosófico, mas, já no século XX, questões postas por abordagens mais sociológicas, 
como as de Max Scheler (1874-1928), Karl Mannheim (1893-1947) e, embora pouco lido na época, Ludwik Fleck (1896-1961) oferecem novas perspectivas para os estudos sobre a dinâmica do conhecimento. Esta fase se fecha, esquematicamente, no final da década de 1930.

Entre os anos de 1940 e 1960, tais estudos tomam expressão mais consolidada com os resultados de pesquisas de Robert K. Merton (1910-2003) e autores influenciados por abordagens marxistas. No período de sua consolidação, nas décadas de 1950 e 1960, os ESCT se caracterizam pelo estudo da estrutura, mudanças e organização da comunidade científica, da cientometria e do papel dos cientistas na sociedade. Destacam-se nomes como Joseph Ben-David, Warren O. Hagstrom e Derek de Solla Price. A instituição científica era o mote de tais estudos e era consenso a alegação de que à sociologia não cabia o estudo do conteúdo do conhecimento gerado.

No final da década de 1970, surgem pesquisas na área, com novos interesses, diferenciando-se dos demais pelas investigações fortemente voltadas às influências do contexto social, na relativa determinação do conteúdo do conhecimento científico. O amadurecimento dos estudos sobre ciência e tecnologia nesta fase que se estende até os dias atuais, é destacado pela institucionalização de grupos de pesquisa, linhas temáticas em programas de pós-graduação, periódicos e congressos especializados e por uma multiplicidade de abordagens. Autores como Bruno Latour, David Bloor, Michel Callon, Barry Barnes, Steven Shapin, Trevor Pinch, Pierre Bourdieu, Karin Knorr-Cetina, Harry Collins, entre outros, representam parte desta nova paisagem dos estudos sociais em ciência e tecnologia. ${ }^{1}$

Poder-se-ia, neste suscinto panorama histórico de emergência e consolidação da sociologia da ciência ou, de forma mais genérica, dos ESCT,

1 Algumas coletâneas são muito importantes para uma introdução destes estudos, tais como: Jasanoff (2004); Johnson e Wetmore (2009); Hackett; Amsterdamska; Lynch; Wajcman (2007); Knorr-Cetina; Mulkay (1992); Law e Hassard (1999); Mackenzie e Wajcman (1999) e Cutcliffe e Mitcham (2001). 
discorrer sobre os principais conceitos, objetivos teóricos e pressupostos metodológicos presentes nas diversas abordagens autorais e as efetivadas em "escolas", ou tradições de grupos e programas de pesquisa, tal como pode ser conferido em Mattedi (2006), Rodrigues (2005) e Martin (2003). Mas este não é o principal objetivo deste texto, contudo, para nuançar esta síntese histórica, dois autores, talvez, mereçam um pouco mais de atenção em alguns pontos, pela influência e controvérsias do conjunto de ferramentas conceituais e metodológicas que carregam consigo. Ademais, tais autores representam as fases distintas destacadas acima, sendo referência em cada uma delas.

O primeiro, Robert Merton, estruturou uma abordagem fundamentada nas normas e valores de conduta da comunidade científica, com seus sistemas simbólicos de recompensa para as pesquisas competentes, criativas e honestas. Sua preocupação, em relação à ciência, é com suas formas de organização e autonomia, grau de institucionalização, desempenho e manutenção e o papel histórico do cientista na moderna sociedade ocidental. As normas e valores que, para Merton (1979, p.37-52), constituem os imperativos morais, fundamentam o ethos da comunidade científica e têm entre seus principais objetivos a ampliação do conhecimento certificado. Estas normas são formadas por preferências, permissões, prescrições e proscrições morais e técnicas, no contexto dos valores de uma instituição, e estabelecem um código informal de orientação da prática de cada cientista e estruturação da ciência enquanto atividade social. Estes imperativos seriam: universalismo, comunismo, ceticismo organizado, desinteresse, originalidade e humildade.

O segundo autor, David Bloor (1991), relativamente mais importante que o primeiro nas perspectivas contemporâneas dos estudos em ciência e tecnologia, deu origem a um fértil e controverso programa de pesquisa que inspirou, de certa maneira, outras abordagens contemporâneas como o Programa Empírico do Relativismo e, de certo modo, a Sociologia da 
Tradução, sobretudo pelo conceito de simetria, um dos princípios do Programa Forte em Sociologia do Conhecimento. Grande parte dos embates em torno de determinadas abordagens dos ESCT deriva de questões epistemológicas (mesmo ontológicas) e metodológicas deste princípio (MATTEDI, 2004). Mas, ao contrário das abordagens tradicionais influenciadas mais diretamente por Robert Merton, o Programa Forte propõe uma investigação sociológica voltada aos processos sociais de elaboração cognitiva e aceitação do conhecimento científico, ao seu conteúdo, às correspondências entre ordem cognitiva e ordem social. Após os anos de 1960/1970, há uma maior objeção na sociologia da ciência, aos estudos que afastavam de seus questionamentos a produção social do conteúdo da ciência, suas teorias, métodos e conceitos. Não bastava mais, contrariamente à tradição mertoniana, ocupar-se apenas dos sistemas de organização social da ciência e de suas estruturas de funcionamento tais como normas, sanções, institucionalização das disciplinas, departamentos e programas de pesquisa.

O conhecimento científico, nesta perspectiva, não estaria livre dos condicionantes sociais, muito pelo contrário, sua validade e legitimidade possuem estreita correlação com as dinâmicas sociais que perpassam o campo científico. Dos quatro princípios programáticos do Programa Forte (BLOOR, 1991, p.7), causalidade, imparcialidade, reflexividade e simetria, este último o mais importante nesta discussão introdutória, pretendia indagar a necessidade de dar tratamento equivalente ao verdadeiro e falso, ao científico e social. Autores como Michel Callon, John Law e Bruno Latour, para citar apenas alguns nomes, ampliam o efeito da noção de simetria ${ }^{2}$. As análises, principalmente para o último autor, passam a basear-se na equivalência e simetria analítica entre humanos e não-humanos nos processos sociotécnicos. Essa equivalência apóia-se em três pontos: 1) a relação de

2 Bruno Latour e sua antropologia simétrica, provavelmente é o autor de maior penetração nas pesquisas acadêmicas brasileiras, no entanto, é ainda pouco usado para o estudo da ciência e mais para a discussão da antropologia contemporânea. 
igualdade, segundo a ordem de importância e valor para uma rede sociotécnica dos humanos, organismos biológicos de qualquer tipo, por exemplo, e objetos materiais; 2) a relação de implicação recíproca ou dificuldade em apontar empiricamente diferenças entre os agenciamentos humanos e não humanos e 3) a complementaridade entre ciência e técnica e a constante tecnificação do cotidiano das sociedades e interações humanas.

O conceito de actante e, mais tarde, ator-rede (LATOUR, 2000, p. 138) aparece neste contexto como ferramenta capaz de dar um tratamento simétrico a uma diversidade de atores, principalmente em ambientes sociotécnicos. A teoria do ator-rede desdobra-se constantemente em meio a críticas e problemas de operacionalização metodológica, mas continua como uma das principais abordagens contemporâneas nos estudos sobre ciência e tecnologia (NEYLAND, 2006). No limite, talvez, e do ponto de vista epistemológico, o que está em questão é a possibilidade de uma descrição do mundo humano — também formado por atores inumanos - não baseada em uma perspectiva radicalmente antropocêntrica. No processo de construção e nomeação de um artefato tecnológico, por exemplo, parte-se geralmente de ensaios experimentais em laboratórios que, aos poucos, vão caracterizando as qualidades esperadas e não esperadas de um produto, bem como seus atributos e sentidos sociais. Estas experimentações mobilizam um conjunto de respostas incluídas em um sistema de provas e considerações metodológicas para a validação do artefato no campo científico ou, seguindo a matriz conceitual da teoria do ator-rede, no interior das redes sociotécnicas. O produto ou objeto antes inexistente vai substantivando-se através de um sistema de representação que envolve uma estreita relação entre equipamentos laboratoriais, teorias, conceitos, agendas de pesquisa, agências de fomento, divulgação científica e debate entre os pares. A ciência e a tecnologia emergem dessas pesquisas, não como provenientes de uma natureza ou 
realidade pré-existente, e sim como realização da prática humana, como um movimento incessante de entrechoques e acomodações entre atores, princípios teóricos e dados empíricos.

Estes programas ou abordagens recentes centralizam suas questões em como o conhecimento científico é dependente do contexto social e, principalmente no caso do modelo ator-rede (LAW, 1989), como o papel do sujeito ou do cientista é atenuado em relação aos agentes inumanos, como os elementos de uma rede adquirem forma e estão dependentes uns dos outros, como se estrutura a mobilização de recursos para a construção de um fato científico e como as entidades que a formam (bactérias, genes, nanopartículas, gases de efeito estufa, por exemplo) adentram nas discussões mais amplas da sociedade ou em suas agendas políticas. As novas abordagens nos estudos sobre ciência, tecnologia e sociedade reforçam o enfoque nas zonas intermediárias ou de confluência entre os aspectos "internos" e as práticas "externas" do conhecimento científico. O que unifica as diversas abordagens e enfoques destes estudos é que todas elas tendem a refletir o conhecimento científico muito mais no âmbito do "mundo da práxis cotidiana" do que nos enfoques mais tradicionais derivados da filosofia da ciência ou das discussões sobre seus determinantes epistemológicos. Ou seja, em como a ciência "deveria ser" e não o que ela "realmente é".

\section{Controvérsias e negociações no centro da produção de teorias e experimentos}

Após o período de institucionalização desse campo de pesquisa, parte da agenda dos estudos CTS logrou mostrar que a construção do argumento verdadeiro de determinada controvérsia científica é muito mais o resultado de negociações, acordos, interpretações e concessões sobre resultados e objetivos da pesquisa (LATOUR, 2005; GARRETY, 1997) do 
que a perfeita representação de um fato natural. A controvérsia é um tipo de ação intrínseca à ciência, principalmente quando o centro da disputa argumentativa envolve conhecimentos ainda não assegurados. É no meio destas incertezas que as decisões são tomadas no processo de estabilização de um conhecimento (CALLON, 2006; CALLON; LASCOUMES; BARTHE, 2001, p. 11-12).

Visualizar com clareza o significado de um resultado não é simples, pois existe muita disputa entre os cientistas sobre as formas de observação de um fenômeno e as maneiras de interpretá-lo. Imagine-se a complexidade de negociação em áreas com maior penetração social, em termos de resultados práticos e divulgação midiática, como as ciências das mudanças climáticas, biotecnologias e nanotecnologias. Entender como se constitui uma evidência científica é alvo cada vez mais corriqueiro em linhas de pesquisa ligadas a abordagens CTS. Citam-se aqui os estudos de Pinch (1985) sobre detecção de neutrinos e a série "O golen", de Collins e Pinch (1998; 2003), em que procuram construir esquemas analíticos sobre o contexto constitutivo da evidência de dados experimentais, apresentando tal contexto como problemático e preenchido de incertezas e desacordos. Estas pesquisas buscam mapear uma região de difícil visualização ${ }^{3}$, situada desde a heterogeneidade epistemológica de processos envolvidos na construção do conteúdo da ciência, até âmbitos mais gerais, relacionados às circunstâncias institucionais, curriculares e de financiamento da pesquisa.

A dificuldade é desatrelar os principais recursos argumentativos e os protagonistas fundamentais do enovelado de questões e fatores secundários envolvidos nas negociações tecnocientíficas, desde o interior dos laboratórios até as mobilizações em faixas mais difusas do contexto social (BROWN, 2009). A flexibilidade interpretativa no processo de construção

3 No caso, uma complexa interação entre elementos sociais e cognitivos que torna sua descrição analítica inviável. 
e uso de tecnologias, ou produção de dados através de ensaios experimentais, é central nas disputas sociotécnicas. Dependendo da correlação de forças dos grupos envolvidos, há a geração de mecanismos de fechamento de controvérsias e a orientação de uma determinada tecnologia ou conhecimento científico a um patamar de verdade e funcionalidade no sistema (COLLINS, 1981; PINCH; BIJKER, 1984) ${ }^{4}$.

Ainda dentro da agenda de negociações, existem linhas de pesquisa voltadas à compreensão das relações entre tradições de conhecimento teórico e experimentais ou, ainda, da sedimentação de interações interpessoais e culturais no âmbito das práticas de pesquisa que propiciam o desenvolvimento de novos campos de investigação. Andrew Pickering (1990 e 1999), por exemplo, em clássico e controverso estudo sobre física de partículas (ou, com destaque na investigação experimental, física de altas energias) argumenta que um conjunto de especulações teóricas pode preparar o terreno para uma tradição experimental obter provas de partículas hipotéticas e atingir novos desdobramentos conceituais e teóricos, em uma sucessão de complementações de problemas e respostas. Em um ambiente científico e tecnológico cada vez mais indissociável, a criação de novas disciplinas e áreas do conhecimento depende, em parte, de disputas internas entre cientistas consolidados e uma nova geração ávida por espaço que, para sair da sombra, necessita marcar posição através da criação de linhas de pesquisas mais especializadas. Estas se tornam novos e arejados temas ou áreas do conhecimento, frutos, muitas vezes, da confluência de disciplinas/convergência tecnológica (relação conhecimento teórico/experimento), tal como foi a bioquímica, a biotecnologia e, atualmente, a nanociência e nanotecnologias, a spintrônica, neuro-

4 Para uma revisão destes estudos com enfoque em tecnologia, especialmente a Construção Social da Tecnologia (Social Construction of Technology ou na sigla SCOT), conferir Bijker (2010). 
ciência, tecnologias da informação e comunicação (TIC), pesquisas em infraestrutura eletrônica para software, recursos computacionais, Internet e sistemas virtuais de simulação e colaboração.

Esta discussão dos estudos CTS ou especificamente da sociologia do conhecimento científico transita entre as ligações das formas de conjugação de dados experimentais - na constituição de modelos da realidade - e sua coerência com os relatos culturais envolvidos no conhecimento teórico hegemônico de um período. A influência de Ludwig Wittgenstein — via Programa Forte e interacionismo simbólico — pode comprovar-se na perspectiva sobre a definição do contexto científico como um universo estruturado pela linguagem e pelos significados compartilhados entre seus agentes. A estruturação dos relatos científicos é inteligível porque existe uma convivência e treinamento dos cientistas a uma linguagem particular, a matemática, por exemplo, e a todo um arcabouço teórico-conceitual moldado sob o aspecto disciplinar ou temas de pesquisa. Nota-se, quanto a este aspecto, influência do trabalho pioneiro de Thomas Kuhn para a mudança de foco de estudo na sociologia do conhecimento, influência assumida explicitamente por Barnes (1982).

O nível de validação entre teoria e experimento representa uma zona histórica de embates. A racionalidade é chamada a arbitrar impasses e contradições entre previsões teóricas e a capacidade experimental de prová-las. Muito mais do que a opinião do cientista individual para a tomada de decisão e arbítrio de um experimento ou dado, é a eficácia de uma agenda de pesquisa e a produção conjunta da racionalidade científica formada pela comunidade ou coletividade de cientistas, que deve ser levada em conta (SOLOMON, 2001). A física atômica e nuclear, a teoria da relatividade especial, o eletromagnetismo, todas estas áreas, historicamente, passaram por muitos acordos privados, embates entre tradições de pesquisa e de carreira, negociação profissional, concessões financeiras 
e políticas. É difícil amarrar todos os pontos desta intriga de bastidores ante um determinado conhecimento, um procedimento técnico, coerente o bastante, entre um nível de validação, para uma maioria apontar a racionalidade de sua aceitação.

\section{A ciência como prática e representação: a dimensão social do laboratório}

A ciência, a partir do que foi dito acima, não é constituída apenas por um conjunto de teorias, mas por uma prática de intervenção sobre o mundo. Advém desta proposição a independência relativa do experimento em relação à teoria. Shapin e Schaffer (2005, p. 56-57) associam três tecnologias à produção do conhecimento e objetos científicos. A primeira delas são as tecnologias materiais, o que forma o aparato laboratorial, os instrumentos ou aparelhos necessários à simulação de fenômenos naturais, à produção de objetos experimentais, teóricos e à criação de procedimentos de utilização destes equipamentos. A segunda são as tecnologias sociais, estas organizam as formas de legitimidade e credibilidade dos resultados e experimentos científicos. Através destas tecnologias estabelece-se a ordem de quem faz parte e como se ordena a comunidade científica, quem pode tecer considerações acerca de um assunto (especialista) e quem não pode (leigo). A terceira são as tecnologias literárias ou tecnologias de inscrição, o modo de representar, através de inscrições, os objetos do conhecimento, é a maneira de fazer circular, com um mínimo de sentido, o conhecimento de algo através de um contexto fora do local e momento de produção (o laboratório, por exemplo). Estas tecnologias elaboram esquemas semióticos de "testemunhas virtuais" para a rede tecnocientífica geral; isto é, sem vinculações diretas com o contexto estrito de produção de determinado conhecimento ou "fato científico". 
O argumento científico, como exposto pelas "três tecnologias" de Shapin e Schaffer (Ibidem), não envolve apenas uma relação cognitiva de mútuo apoio entre teoria e manipulação experimental, mas uma série de fatores tais como experiência acadêmica, tipos de equipamentos empregados no experimento e a rede institucional à sua volta, métodos utilizados, acesso a recursos materiais e conceituais, o periódico no qual se publica, quem publica e outros capitais científicos. A política, neste sentido, não é um evento exterior à ciência e que obsta sua prática. A agonística científica na busca de convencimento e justificação utiliza-se de um repertório protocolar para criar um mínimo de entendimento e tolerância. Este repertório baseia-se no experimento reprodutível de forma disciplinada, com equipamentos precisos e especializados e no seu testemunho público, feito no laboratório (KNORR-CETINA, 1999, p. 28-33). A ciência moderna procura, através da prática experimental, dar voz às coisas, busca no testemunho fenomênico a verdade, já que o simples uso da palavra é caro, demanda mais tempo e habilidade do que a construção e demonstração experimental. Neste sentido, a prática do experimento na geração do conhecimento pode ser compreendida como uma convenção, um processo argumentativo para o assentimento. Deste ponto de vista, um problema de ordem política.

O contexto sociopolítico está sempre influenciando a prática científica de algum modo, e não há constatação empírica de cientistas buscando uma verdade unicamente pelo desejo de conhecê-la, tendo em vista o nível de influências e complexidade da ciência e tecnologias atuais. Mas, mesmo a ciência sendo influenciada por "contextos externos" e por interesses não estritamente cognitivos, ela avança, resolve problemas, justamente por ajustar interesses sociais e cognitivos. Ou seja, a ciência bem sucedida é aquela que soube utilizar o "social" a seu favor, teve meios de ajustar múltiplos interesses sociais e políticos no desenvolvimento e reso- 
lução de problemas cognitivos, finalmente, aquela sustentada por uma rede sociotécnica estável.

Em ciência, a noção de representação precisa manter um efeito bem-sucedido de correspondência entre uma teoria e um domínio de fenômenos do mundo. Evidentemente, a ciência e sua diversidade de métodos e procedimentos, têm suas formas de produzir esta relação. Muito da eficiência desta relação está em produzir valores cognitivos efetivos, ou seja, uma adequação entre dados empíricos e teoria, entre outras teorias e a inexistência de hipóteses ad hoc. Para a tradição sociológica subjacente aos estudos sociais em ciência e tecnologia, este processo não se dá pela legitimidade conferida à natureza pela pesquisa em si, mas a um feliz esforço de uma prática social específica: a dinâmica da prática científica ${ }^{5}$. As divergências criadas quando o assunto se volta à tentativa ou negativa de replicar um experimento para validar uma posição como a do consumo de alimentos geneticamente modificados ou aquecimento global, é extremamente difícil de ser formalizada em torno de regras (COLLINS, 1992, p. 38-46) que encerrem a validade de um resultado experimental e suas induções conceituais e teóricas.

Se os objetivos acima tendem a parecer adequados a uma escala relativamente ampla de análise, vale ressaltar que a maior parte das conclusões dos autores até agora citados não se pauta por abordagens cujas unidades sociais de análise se caracterizam por parâmetros meso e macrossociais. Através da ênfase na perspectiva microssocial, centrada principalmente nos estudos de laboratório e nas múltiplas e localizadas controvérsias sobre interpretação da realidade e produção de consensos (COLLINS, 1992; LATOUR e WOOLGAR, 1997), estas abordagens buscam analisar também

5 Disto resulta a relação dos ESCT com a teoria social contemporânea, que valoriza a ação prática nas análises. Para esta discussão, ver Schatzki; Knorr-Cetina; Savigny (2001), ou para o que eles chamam de pratical turn na teoria social contemporânea. 
a prática laboratorial, o processo mesmo da produção e estabilização de controvérsias do conhecimento científico ou a formação de consensos sobre determinados fatos e proposições cognitivas. O que se convencionou chamar de etnografia densa de laboratório procura enfatizar o que diferencia a produção científica e tecnológica de outras formas de conhecimento ${ }^{6}$, como os agentes da ciência manipulam objetos, realizam experimentos e refletem sobre esta prática e, principalmente, como o "social" se insere neste tipo de conhecimento que deseja, por vezes, apresentar-se como liberto de qualquer contrapartida ou influência social.

Estas reflexões, contudo, não se restringem apenas aos laboratórios, pois suas ferramentas conceituais e analíticas fornecem pistas para se compreender a concatenação dos processos sociotécnicos sociedade afora. A importância das ciências humanas nas discussões sobre produção científica, tecnológica e inovação é que, cada vez mais, a tecnociência enreda-se tanto na formação de subjetividades identificadas com uma cultura tecnológica, quanto nas ações humanas diárias envoltas pela produção e interação incessantes de entidades tecnológicas.

Para entender a lógica do argumento científico quanto aos usos sociais das tecnologias e da ciência é necessário analisar a dinâmica de construção dos fatos científicos e a estruturação de seus regimes de enunciação (o quanto há de mediação social na construção das verdades dos fatos científicos). A questão que se põe é o quanto o mundo social é exógeno à produção científica e tecnológica. Assume-se aqui uma postura moderada e heterogênea (HESS, 1997, p. 82) do construcionismo social na sociologia do conhecimento científico, ou seja, o social configura certos aspectos dos conteúdos da ciência e tecnologia. As variáveis sociais

6 Este empenho em deslindar diferenças de conhecimentos não é a mesma coisa que explicar a suposta autonomia do conhecimento científico em relação ao social, ao político ou a outras instâncias da sociedade. 
constituem e interferem em padrões de escolhas sobre pesquisas, seus temas e design teórico-interpretativo, o contexto mais específico da prática científica. Esta prática confere uma estrutura e um sentido na composição da realidade, o mundo não está passivo à sua descoberta. Este construcionismo moderado ${ }^{7}$ aceita um mundo real, mas moldado por interesses e variáveis sociais e objetivado por teorias. Estas enquanto sistemas explicativos da realidade, codificados por limites culturais (linguístico, por exemplo) e categorias e valores socioculturais. Não se consideram os interesses sociais e culturais como elementos negativos nas teorias, desde que estes elementos e o pertencimento social dos agentes da ciência estejam colocados como arbitrários e, também, como variáveis objetivadas e não ignoradas através de algum artifício utópico de neutralização.

As representações sobre natureza passam necessariamente por codificações provenientes de aspectos e significados culturais e político-culturais incluídos como parte integrante da episteme das teorias científicas. A opção por um "construcionismo heterogêneo", por marcar posições sociais e cognitivas no processo de construção do conhecimento científico e tecnológico, relaciona-se a uma perspectiva de estudo em que os conteúdos da ciência e tecnologia são construídos em conjunto e progressivamente nas estruturas e interações sociais.

Considerações finais

Como foi visto, três grandes objetivos conectados entre si podem ser enumerados nas pesquisas relacionadas aos estudos sociais em ciência e tecnologia. O primeiro objetivo refere-se às pesquisas que buscam o "social" no conhecimento científico, inclusive em seu conteúdo; o segundo busca identificar os múltiplos interesses ligados aos grupos sociais que

7 Para efeitos de comparação, Latour e Woolgar (1997) e Pickering (1999) sustentam uma postura mais radical de construcionismo do que a aqui almejada, embora cada caso tenha suas particularidades quanto à relação da sociedade em geral e a estabilização de fatos científicos. 
compõem as redes tecnocientíficas e, por último, têm-se os estudos que procuram analisar as disputas e legitimações decorrentes das definições de problemáticas e enunciados científicos, como apresenta Callon (2006), em pequeno e emblemático artigo. Estes objetivos são geralmente pautados por uma análise pormenorizada do contexto sociocultural de emergência de uma disputa ou "fato científico e tecnológico", e da posterior dinâmica das redes sociais que sustentam este fato.

A ciência, para os ESCT, não é um conjunto uniforme de conhecimentos, independente de contextos específicos de produção e circulação. Contextos materiais e discursivos são instâncias indissociáveis do conhecimento. Isso esclarece o motivo da impossibilidade de se entender e reproduzir certos experimentos apenas com teorias, relatórios e manuais; é necessário todo um aparato material e razoável domínio dos processos de inscrição feitos entre a "natureza" e o suporte teórico das demonstrações científicas.

A produção do conhecimento científico e tecnológico, grosso modo, é constituída por um apanhado de problemas e questões materiais, tais como o aparato laboratorial, os instrumentos necessários à simulação de fenômenos naturais, a produção de objetos experimentais e teóricos e a criação de procedimentos destes aparelhos e instrumentos. Estes elementos materiais só funcionam quando operacionalizados junto a questões e problemas sociais, tais como as formas de legitimidade e credibilidade dos resultados e experimentos científicos. Estas questões ajudam a pensar o estabelecimento da ordem social, como se institui uma rede científica e/ou de especialistas, quem pode tecer considerações acerca de um assunto especializado e quem não pode. E, por último, existem as questões e problemas ligados aos modos de representar e divulgar os objetos do conhecimento, tanto aos especialistas, como ao público leigo.

As áreas temáticas abertas pelo conhecimento científico e inovações tecnológicas são de grande interesse para as ciências humanas. Há 
muito que explorar na junção entre artefatos tecnológicos e relações sociais. A dinâmica tecnocientífica materializa novas configurações de mundo, e é no rastro das relações de poder, das reconfigurações sociais e tecnológicas, dos interesses envolvidos nas mudanças tecnológicas, da construção de novas sensibilidades e identidades que as ciências humanas entram como ferramental interpretativo, na busca de análises mais apuradas desta expansão dos objetos tecnológicos para além do espaço laboratorial.

As tecnologias e os discursos são manifestações indissociáveis, formam um entrelaçamento de imagens e objetos técnicos que se complementam em termos de efetividade. É na formação discursiva que o objeto adquire seu sentido de uso e mesmo suas possibilidades de transformação. Uma inovação consolidada, assim, não é pura engenhosidade técnica, mas a integração de elementos comerciais, políticos, ambientais, culturais à sua lógica de implementação: quanto mais bem amarrados estes elementos heterogêneos, mais efetiva e convincente a consolidação de um sistema tecnológico (MACHADO, 2004, p. 64-65). O fenômeno de rotinização das inovações - conjunto de elos entre capital, ciência e indústria - modifica radicalmente as representações de natureza e humanidade, as interações entre coletivos humanos e não humanos. A flexibilidade e diversidade de uso e aproveitamento dos objetos técnicos, dos variados maquinários e equipamentos provocam um aumento considerável do mundo artificial, relevando sua importância ontológica e política frente ao mundo humano. Já que as ciências humanas não participam ativamente da produção laboratorial, têm, ao menos, o papel de tornar pública a rotina de produção dos artefatos tecnológicos, dos interesses envolvidos, das tendências de conjunção da tecnologia, da produção discursiva e dos possíveis impactos socioambientais provenientes da sua disseminação. 


\section{Social studies in science and technology and its different approaches}

\section{Abstract}

This short introduction to Social Studies in Science and Technology (SSST) intends to present the usual issues and the different theoretical approaches that comprise this still new research field. It is sought to provide an outlook on the disputes, both external to this field - between sociologists and epistemologists and internal to it, between distinct views on science and technology that concern, today, sociologists, anthropologists and political scientists within this rich scene of social research.

Key words: Knowledge. Science. Technology. Symmetry.

\section{Referências}

BARNES, Barry. T. S. Kuhn and the Social Science. Nova York: Columbia University Press, 1982.

BIJKER, Wiebe E. How is Technology Made?-That is the Question! Cambridge Journal of Economics, vol. 34, n. 1, p. 63-76, 2010.

BLOOR, David. Knowledge and Social Imagery. Chicago: the University of Chicago Press, 1991.

BROWN, Mark B. Science in Democracy: Expertise, Institutions and Representation. Cambridge, Massachusetts: MIT Press, 2009.

CALLON, Michel; LASCOUMES, Pierre; BARTHE, Yannick. Agir dans un monde incertain: essai sur la démocratie technique. Paris: Seuil, 2001.

CALLON, Michel. Luchas y negociaciones para definir qué es y qué no es problemático. La socio-lógica de la traducción. Redes: Revista de Estudios Sociales de la Ciencia, vol. 12, n. 23, p. 103-128, 2006.

COLLINS, Harry M. Changing Order: Replication and Induction in Scientific Practice. Chicago: University of Chicago Press, 1992.

COLLINS, Harry; PINCH, Trevor. O golen: o que você deveria saber sobre ciência. São Paulo: UNESP, 2003. 
COLLINS, Harry; PINCH, Trevor. The Golem at Large: What you Should Know about Technology. Cambridge: Cambridge University Press, 1998.

COLLINS, Harry. Introduction: Stages in the Empirical Programme of Relativism. Social Studies of Science, vol. 11, n. 1, p. 3-10, 1981.

CUTCLIFFE, Stephen H. e MITCHAM, Carl (Eds.). Visions of STS: Counterpoints in Science, Technology and Society Studies. Albany, NY: State University of New York Press, 2001.

GARRETY, Karin. Social Worlds, Actor-Networks and Controversy: The Case of Cholesterol, Dietary Fat and Heart Disease. Social Studies of Science, vol. 27, n. 5, p. 727-773, 1997.

HACKETT, Edward J.; AMSTERDAMSKA, Olga; LYNCH, Michael e WAJCMAN, Judy (Eds.). The Handbook of Science and Technology Studies. Cambridge, MA and London: MIT Press, 2007.

HESS, David. Science Studies: an Advanced Introduction. New York/London: New York University Press, 1997.

JASANOFF, Sheila (Ed.). State of knowledge: The co-production of science and social order. Cambridge: Harvard University Press, 2004.

JOHNSON, Deborah G. e WETMORE, Jameson M. Technology and society: Building our Sociotechnical Future. Cambridge, MA: The MIT Press, 2009.

KNORR-CETINA, Karin. Epistemic Cultures: How the Sciences Makes Knowledge. Cambridge: Harvard University Press, 1999.

KNORR-CETINA, Karin; MULKAY, Michael. (Eds.). Science Observed: Perspectives on the Social Study of Science. London: Sage, 1992.

LATOUR, Bruno e WOOLGAR, Steve. A vida em laboratório: a produção dos fatos científicos. Rio de Janeiro: Relume Dumará, 1997.

LATOUR, Bruno. Ciência em ação: como seguir cientistas e engenheiros sociedade afora. São Paulo: Editora UNESP, 2000.

. Reassembling the Social: An Introduction to Actor-Network Theory. New York: Oxford University Press, 2005.

LAW, John. Le laboratorie et ses réseaux. In: CALLON, Michel. La science et ses réseaux: gênese et circulation des faits scientifiques. Paris: Éditions La Découverte/ Unesco; Strasbourg: Conseil de l'Europe, p. 117-148, 1989.

LAW, John; HASSARD, John (Eds.). Actor Network Theory and After. Oxford: Blackwell Publishing, 1999.

LYNCH, Michael. Act and Artifact in Laboratory of Science. London: Routledge, 1985. 
MACHADO, Carlos José S. Tecnologia, meio ambiente e sociedade: uma introdução aos modelos teóricos. Rio de Janeiro: E-papers, 2004.

MACKENZIE, Donald; WAJCMAN, Judy (Eds.). The Social Shaping of Technology. Buckingham: Open University Press, 1999.

MARTIN, Olivier. Sociología de las ciencias. Buenos Aires: Nueva Visíon, 2003. MATTEDI, Marcos. Dilemas da simetria entre contexto social e conhecimento: a redefinição das modalidades de abordagem sociológica do problema do conhecimento. Política \& Sociedade, Florianópolis, n. 4, p. 41-79, 2004.

. Sociologia e Conhecimento: introdução à abordagem sociológica do problema do conhecimento. Chapecó, SC: Editora Argos, 2006.

MERTON, Robert K. Os imperativos institucionais da ciência. In: DEUS, Jorge D. de (Org.). A crítica da ciência: sociologia e ideologia da ciência. Rio de Janeiro: Zahar Editores, p. 37-52, 1979.

NEYLAND, Daniel. Dismissed Content and Discontent: An Analysis of the Strategic Aspects of Actor-Network Theory. Science, Technology \& Human Values, vol. 31, n.1, p. 29-51, 2006.

PICKERING, Andrew. Knowledge, Practice and Mere Construction. Social Studies of Science, vol. 20, n. 4, p. 682-729, 1990.

. Constructing Quarks: A Sociological History Of Particle Physics. Chicago: University of Chicago Press, 1999.

PINCH, Trevor. Towards an Analysis of Scientific Observation: The Externality and Evidential Significance of Observational Reports in Physics. Social Studies of Science, vol. 15, n. 1, p. 3-36, 1985.

PINCH, Trevor J.; BIJKER, Wiebe E. The Social Construction of Facts and Artefacts: or How the Sociology of Science and the Sociology of Technology Might Benefit Each Other. Social Studies of Science, vol. 14, n. 3, p. 399-441, 1984.

RODRIGUES, Léo P. Introdução à sociologia do conhecimento, da ciência e do conhecimento científico. Passo Fundo: UPF Editora, 2005.

SCHATZKI, Theodore; KNORR-CETINA, Karin; SAVIGNY, Eike V. (Eds.). The Practice Turn in Contemporary Theory. London; New York: Routledge, 2001.

SHAPIN, Steven; SCHAFFER, Simon. El Leviathan y la bomba de vacuo: Hobbes, Boyle y la vida experimental. Bernal: Universidad Nacional de Quilmes Editorial, 2005.

SOLOMON, Miriam. Social Empiricism. Cambridge, MA: MIT Press, 2001. 